\title{
CLIMATE Clinical Help System
}

\author{
Rini Hoogkamer and Martin Briscoe
}

Product CLIMATE Clinical Help System v1.0 Hardware: PC running windows 3.1

Price: $\$ 25$

Author: Prof. Gavin Andrews, Australia

The CLIMATE (CLnical MAnagement and Treatment Environment) clinical help system is a pre-release component of what will be an integrated Information System, based on the World Health Organization's Primary Health Care version of ICD-10. It was written by Professor Gavin Andrews and his team at the Clinical Research Unit for Anxiety Disorders, Darlinghurst, NSW Australia, and presented as a "multi-faceted system that will faclitate and promote modern principles of good patient care".

\section{About the programme}

CLIMATE is essentially a computerised book containing 200 to 300 screens of information covering the assessment, diagnosis and management of psychiatric illness. The assessment chapters contain flow charts for presenting problems and new cases, a problem screening checklist and about 30 screens on taking a psychiatric history and examining the mental state. The diagnostic chapters give the clinical features and differential diagnosis for $\mathbf{2 0}$ of the major ICD-10 categories. The management chapters provide essential information for patients and families; specific counselling for patients and families; medication, and when to seek a specialist opinion for each of the diagnostic categories.

There is an extenstve glossary which expands on the main body of the text, a complete index and an ability to print out information in the form of fact sheets.

CLIMATE works under Windows, thus making it a user friendly programme. Virtually all tasks can be completed by just moving a cursor around the screen using a hand held pointer called a mouse. Clicking one of the buttons on the mouse activates whatever is underneath the screen cursor. The programme displays the text on the screen and gives the user the ability to move instantly from any word in the index to a page containing that word. The same can be done with highlighted words in the text, for example, under the differential diagnosis of dementia clicking the mouse button while the screen cursor is over the word delirium will instantly display the diagnostic criterla for delirium. The text can be annotated with the user's own comments and its pages can be copied into another document.

\section{Who is it for?}

As it stands CLIMATE would be very useful for Community Mental Health Team workers, primary care professionals, trainee psychiatrists and medical students.

\section{Postive features}

CLIMATE comes on a single disk and is easily installed onto a computer. The programme is professionally presented and people used to Windows will instantly feel at home. It contains very useful and clearly presented material, much of which could make excellent handouts for study or for use by patients. One example would be a leaflet on relaxation, its use and how-to-do-it. The programme could prove a useful adjunct in MRCPsych preparation. The glossary has many basic definitions. The information on history taking, psychopathology and management would be very helpful when preparing for clinical presentations.

\section{Drawbacks}

We have only minor niggles about the programme. On every screen an inch was taken up by the CLIMATE logo. Reducing the size of this logo would not detract from the programme. Although the system allows you to make annotations, these are tagged to the beginning of a subject rather than immediately opposite the text to which the annotation 
refers. This is a problem with all Windows help files and one that in our opinion is worth putting up with as using alternative software would very much increase the price of the product.

\section{Overall impreseion}

CLIMATE could be a valuable clinical tool for primary care professionals and those interested in computerised aids for community psychiatry. It will also be a valuable educational aid for medical students and trainee psychiatrists preparing for MRCPsych exams and as such it certainly deserves a place on the shelves of training-scheme libraries.

The CLIMATE Clinical Help System would appear to be a forerunner of a time when computer applications will assist us in our daily clinical practice. As such it is certainly a simple, yet useful, and non-frightening introduction into what can be done with computer technology in psychiatry.

At $\$ 25$ it represents good value for money. Coples of CLIMATE can be obtained from: CLIMATE Promotion, Longman Cartermill Limited, Technology Centre, St Andrews, Fife KY16 9EA, Scotland. Tel: 01334-477660. Fax: 01334-477180.

*Rini Hoogkamer, Senior House Officer in Psychiatry. Wonford House Hospital, Exeter EX2 5AF, and Martin Briscoe, Chairman, Computers in Psychiatry Special Interest Group. Wonford House Hospital, Exeter

"Correspondence

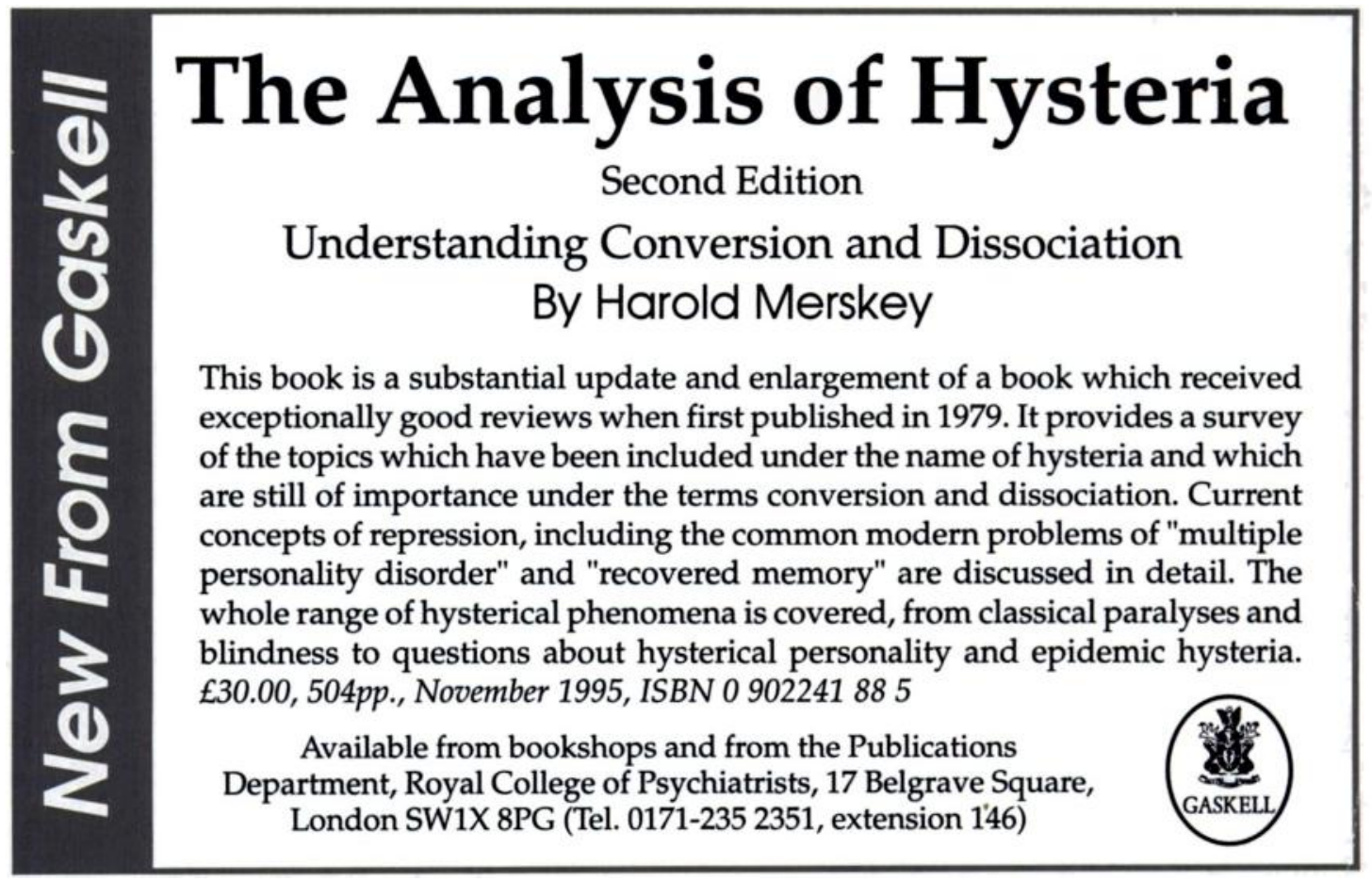

